\title{
OCCLUSION FOR AMBLYOPIA: A COMPREHENSIVE SURVEY OF OUTCOME
}

\author{
F. HISCOX, N. STRONG, J. R. THOMPSON, C. MINSHULL, G. WOODRUFF \\ Leicester
}

\section{SUMMARY}

The results of a long term follow up of all patients from a single health district started on occlusion for amblyopia in 1983 are reported.

Three hundred and sixty-eight patients started treatment, their average age was four years seven months, the average amount of daily occlusion was 1.5 hours and the average length of follow up was 31 months. $37 \%$ of cases achieved a final visual acuity of $6 / 9$ or better and another $33 \%$ a visual acuity of $6 / 12$ or $6 / 18.23 \%$ did not achieve $6 / 18$ and treatment of these patients was regarded as failure. Data for the remainder $(7 \%)$ were incomplete.

The success rate of occlusion treatment varied little with the age of starting treatment. The group with combined strabismus and anisometropia responded least well to treatment.

Occlusion of the non amblyopic eye has long been the mainstay of amblyopia therapy, its use dating back to the 18th century. ${ }^{1}$ There is an extensive literature concerning the results of occlusion therapy, in which authors have reported widely differing success rates ranging between $30 \%{ }^{2}$ and $92 \%{ }^{3}$. This large variation in outcome can be explained by the equally wide variation in methodology adopted in these studies. These differ in the means by which patients are selected (and excluded), the ages of patients treated, the criteria adopted for a diagnosis of amblyopia, the type of occlusion employed, the extent of follow up and the definition of 'success'.

The outcome of any study of amblyopia therapy will suffer bias if patients selection is non-random, and this is particularly the case if, as is frequently done, those who do not comply or attend are excluded. In fact we are unaware of any series of treated amblyopia patients in which the patients were recruited in a representative way from the general population, and in which the results are reproted without exclusions.

In an attempt to investigate the treatment of a repre-

Correspondence to: Ms F. Hiscox, Department of Ophthalmology, Clinical Sciences Building, Leicester Royal Infirmary, PO Box 65, Leicester, LE2 7LX. sentative cross-section of children we have retrospectively identified a cohort of children referred for amblyopia treatment in Leicestershire during a single calendar year. Their outcomes are presented without making any exclusions for age, aetiology, initial vision or lack of compliance.

\section{METHODS}

We retrospectively identified all patients newly referred to the Orthoptic Department in Leicestershire during 1983. The records of these patients were examined and all those prescribed occlusion treatment for amblyopia were identified and included in our cohort. The epidemiological features of this cohort of patients have been reported elsewhere. ${ }^{4}$ We believe that it includes the vast majority of children resident in Leicestershire who started treatment for amblyopia in that year.

The orthoptic case sheets were examined for the following information, which was entered onto a database: date of birth, diagnosis, age of onset, refractive error, initial visual acuity, visual acuity at subsequent visits, amount of occlusion recommended by the orthoptist at each visit, reason for stopping occlusion, final visual acuity and date of discharge. Initial visual acuity was defined as the earliest visual acuity recorded, so long as this was obtained no later than the third visit. If a visual acuity was not obtained until later than this then these data were recorded as not measured. The patients' records were examined up to the end of 1989.

The patients were classified into four different diagnostic groups: strabismic amblyopia, strabismic amblyopia with anisometropia, anisometropic amblyopia and stimulus deprivation amblyopia. Anisometropia was defined as a difference of 1.00 dioptre or more of either sphere or cylinder between the two eyes. For the purpose of amblyopia classification strabismus was defined as manifest strabismus evident on cover testing. The stimulus deprivation group was defined as patients with amblyopia due to lack of adequate stimulus in early life.

All patients treated during this study period were man- 
aged with part-time total occlusion using adhesive eye patches. In addition seven patients were also treated with atropine penalisation, 16 patients with partial occlusion (blenderm on their spectacle lens) and 34 patients with full-time total occlusion for part of their treatment.

We categorised the results of treatment into three groups:

(1) if the final visual acuity in the amblyopic eye was greater than or equal to $6 / 9$,

(2) if the final visual acuity was $6 / 18$ or $6 / 12$,

(3) a visual acuity of $6 / 24$ or less.

Those patients for whom no final vision was recorded or available were classified as 'missing data'.

Our results are based upon the visual acuity obtained on the visit when occlusion treatment was finally discontinued and are presented according to aetiology, initial vision, age of commencing treatment, and the amount of occlusion prescribed.

The chi-squared test is used to compare the pattern of the three outcomes in different groups of children.

\section{RESULTS}

A total of 368 patients commenced treatment for amblyopia in 1983 in Leicestershire. The Leicester Royal Infirmary's Orthoptic Department was responsible for the treatment of all these patients, although 114 (31\%) were treated at outlying clinics.

Manifest strabismus including those with microtropia without identity was diagnosed in $295(80 \%)$ of the cohort. Anisometropia was diagnosed in 142 (39\%) of the children.

The average age of the cohort at first visit to the Orthoptic Department was four years seven months. Table I shows the age of first visit to the Orthoptic Department for each type of amblyopia. The average ages of each of the two groups with strabismus are over two years lower than that of the group with anisometropia and no manifest strabismus.

The means by which initial and final visions were obtained are given in Table II. Two hundred and two (73\%) were tested by the same type of test on both occasions. Two hundred and eighty $(76 \%)$ had linear or Snellen acuity as the final test.

Table III shows the outcome of amblyopia treatment.

Table I. The age at first visit by type of amblyopia, and the average age of the patient according to each type

\begin{tabular}{|c|c|c|c|c|c|}
\hline & \multirow{2}{*}{$\begin{array}{l}\text { Strabismic } \\
\text { \& Aniso- } \\
\text { metropic }\end{array}$} & \multirow[b]{2}{*}{$\begin{array}{c}\text { Aniso } \\
\text { metropic }\end{array}$} & \multirow[b]{2}{*}{ Other } & \multirow[b]{2}{*}{ Total } \\
\hline $\begin{array}{l}\text { Age } \\
\text { Group }\end{array}$ & Strabismic & & & & \\
\hline $0-2$ yrs & 76 & 22 & 0 & 0 & 98 \\
\hline $3-4$ yrs & 80 & 28 & 7 & 2 & 117 \\
\hline $5-7$ yrs & 50 & 19 & 45 & 3 & 117 \\
\hline $8+$ yrs & 13 & 7 & 14 & 2 & 36 \\
\hline Total & 219 & 76 & 66 & 7 & 368 \\
\hline $\begin{array}{l}\text { Mean Age } \\
\text { (months) }\end{array}$ & 48 & 53 & 82 & 77 & 55 \\
\hline
\end{tabular}

Overall $37 \%$ achieved $6 / 9,70 \% 6 / 18$ or better and in $7 \%$ the data were incomplete. Considering only those children for whom the outcome is known, $40 \%$ achieved $6 / 9$ and $75 \%$ achieved $6 / 18$ or better.

Figure 1 shows the outcome of treatment as a percentage according to the age at the start of treatment. This shows that the proportion of children achieving $6 / 9$ or better and also the proportion achieving $6 / 18$ or better was similar in all age groups $(\mathrm{X} 2=5.0, \mathrm{df}=6, \mathrm{p}=0.54)$. Thus $49 \%$ of 75 patients aged $0-2$ years, $76 \%$ of 115 children aged 3-4 years, $78 \%$ of 116 aged $5-7$, and $75 \%$ of 36 aged $8+$ years achieved $6 / 18$ or better. Children who were discharged before a subjective visual acuity was obtained have not been assigned an outcome. The slightly poorer results in the lowest age group may be explained by the fact that most of the missing data were from this group $(23 / 26)$.

Figure 2 shows the outcome of treatment according to the type of amblyopia. The difference is statistically significant $(\mathrm{X} 2=11.5, \mathrm{df}=4, \mathrm{p}=0.02)$. Excluding the patients for whom no final vision was obtained, $6 / 18$ or better was achieved by $78 \%$ of 196 patients in the strabismic group, $60 \%$ of 73 patients in the strabismic with anisometropia group, and $82 \%$ of 66 in the anisometropic group. Twentythree of the 26 patients not assigned an outcome came from the strabismic group. There were only seven patients with amblyopia due to a cause other than strabismus or anisometropia, and all of these achieved $6 / 18$ or better.

The results categorised by initial vision are shown in Figure 3. Subjective visual acuity was not performed on $65(18 \%)$ patients prior to starting treatment and these have been excluded. Only $13 \%$ of patients with an initial vision of less than $6 / 60$ achieved $6 / 18$. Although only $17 \%$ of the 95 patients with an initial vision of between 6/24 and $6 / 60$ achieved $6 / 9$, a further $38 \%$ achieved $6 / 12-6 / 18$, so that a total of $55 \%$ had some benefit from treatment. In the 6/18-6/12 initial vision group, $50 \%$ of the 135 patients achieved $6 / 9$, and surprisingly, of the 50 patients who received occlusion therapy when their initial visual acuity was $6 / 9$ or better, a level at which the diagnosis of amblyopia is questionable, only $82 \%$ achieved a final result of 6/9 or better.

The outcome of treatment is likely to be affected by the compliance of the patients to treatment. With the data available it is not possible to give a definitive measure of compliance with the prescribed amount of occlusion. However the reason for stopping occlusion was recorded. Table IV shows the outcome classified by the reason for stopping occlusion, and shows that the proportion failing to achieve $6 / 18$ was highest (37\%) in the group whose

Table II. The method by which visual acuity was assessed at the initial visit and at the end of the treatment

\begin{tabular}{lccc}
\hline Initial test & \multicolumn{3}{c}{ Final test } \\
\hline & Singles or & Linear or \\
pictures & Snellen & None \\
Singles/pictures & 44 & 82 & 0 \\
Linear/Snellen & 1 & 176 & 0 \\
None & 17 & 22 & 26 \\
\hline
\end{tabular}


Table III. The outcome of amblyopia treatment in our cohort of 368 children

\begin{tabular}{lccc}
\hline Outcome & $\begin{array}{c}\text { Number of } \\
\text { patients }\end{array}$ & Cohort & $\begin{array}{c}\text { Percentage of } \\
\text { known outcomes }\end{array}$ \\
\hline 6/9 or better & 137 & $37 \%$ & $40 \%$ \\
6/12 or 6/18 & 120 & $33 \%$ & $35 \%$ \\
6/24 or less & 85 & $23 \%$ & $25 \%$ \\
Missing data & 26 & $7 \%$ & - \\
\hline Total & 368 & $100 \%$ & $100 \%$ \\
\hline
\end{tabular}

occlusion was stopped due to non-compliance or failure to attend the department. However this group only represents a small proportion $(15 \%)$ of the total cohort; the 21 patients who did not achieve 6/18 and were non-compliant represent $25 \%$ of patients in this outcome group. If all 33 non-compliant patients are removed from the analysis the overall visual results were (1) $6 / 9$ or better achieved by $41 \%$, (2) $6 / 12$ or $6 / 18$ achieved by $32 \%$, (3) $6 / 24$ or less achieved by $21 \%$, and missing data $6 \%$.

The average amount of occlusion prescribed was $1 \frac{1}{2}$ hours daily, with a range between 10 minutes to eight hours occlusion daily. The average duration of active treatment was eight months with a range from one month to 43 months. Figure 4 shows the outcome of treatment according to the total amount of occlusion in hours that was recommended over the period of treatment. It can be seen that the outcome of treatment was best in the patients who had the least occlusion: of the 114 patients who had up to 100 hours of occlusion recommended, $61 \%$ achieved 6/9. The success rate reduces as the total number of hours increases, and only $19 \%$ of the 95 patients who had more than 500 hours of occluson recommended achieved 6/9. These differences are highly significant $(\mathrm{X} 2=50.2, \mathrm{df}=6$, $\mathrm{p}>0.0001)$. The total number of hours recommended ranged from 14 hours to a staggering 3,167 hours.

\section{DISCUSSION}

Previous surveys of amblyopia treatment have reported widely varying success rates. Scott and Dickey ${ }^{3}$ reported a

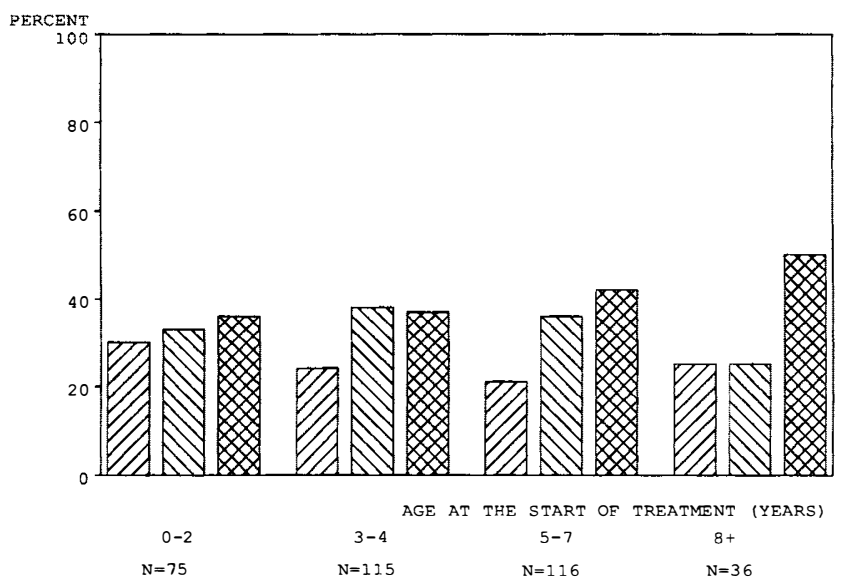

Fig. 1. The percentage outcome of treatment according to age (in years) at the start of treatment. Cross hatching denotes $6 / 9$ or better, falling diagonal hatching denotes $6 / 12$ or $6 / 18$ and rising diagonal hatching denotes less than 6/18.
92\% success rate for a series of 130 patients with strabismic amblyopia who received full-time total occlusion, the majority of whom commenced treatment before the age of six years. However $30 \%$ of these patients were excluded from the final analysis.

The largest survey of outcome of amblyopia treatment was carried out at Sydney Eye Hospital ${ }^{5}$ where 949 cases from a 15 year period were reviewed retrospectively. This was a hospital based study in which it was not possible to be sure that a representative sample of amblyopes was treated. $65 \%$ achieved what was considered a satisfactory result of improvement of the amblyopic eye to within one line of the fixing eye.

Flynn and Cassady ${ }^{2}$ reported a much lower success rate of $30 \%$ achieving a visual acuity of $6 / 12$ or better. Over half of their patients had previously been treated and were regarded as occlusion failures, and $30 \%$ were not offered treatment, or disappeared from follow up.

Bremner ${ }^{6}$ has reported the results of treatment of a group of amblyopes selected at random during one year (1981). Her results showed a $62 \%$ success rate but were limited to children four years of age and older. The children in her series were initially treated with the CAM vision stimulator followed by conventional part-time occlusion with patches.

A recent study from Sweden ${ }^{7}$ gives results that appear to be much better than our own. Their highly successful results have been achieved using a small number of patients, and compliance has been cited as a major factor in their success. The group was studied prospectively by one ophthalmologist and a nurse, with intensive follow up. It might be difficult to maintain the same level of motivation and compliance in routine practice.

Our results are representative of a well defined geographical region and are presented without making any exclusions for age, aetiology, initial vision or lack of compliance.

The difficulty of testing visual acuity in children makes both the diagnosis of amblyopia and the assessment of the

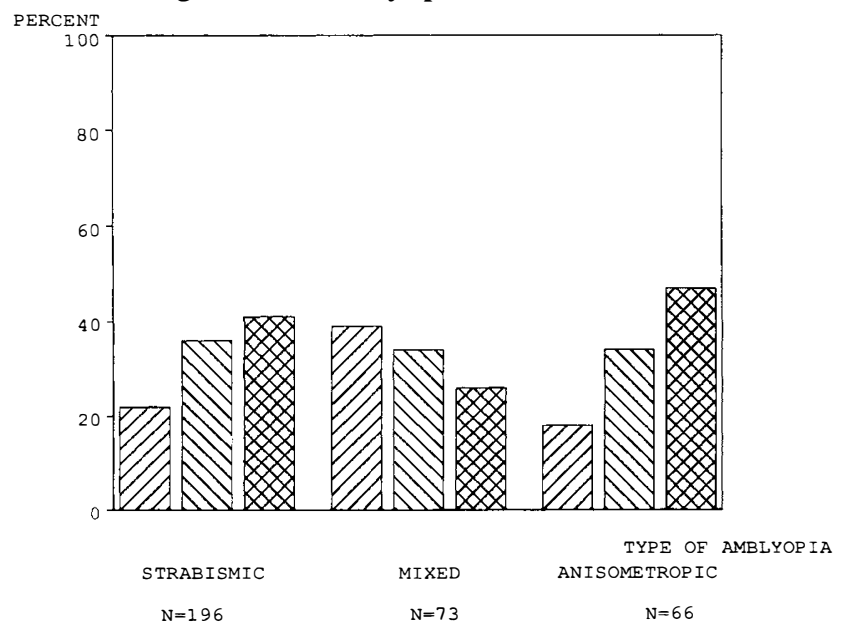

Fig. 2. The percentage outcome of treatment according to the type of amblyopia. Cross hatching denotes $6 / 9$ or better, falling diagonal hatching denotes $6 / 12$ or 6/18 and rising diagonal hatching denotes $6 / 24$ or less. 


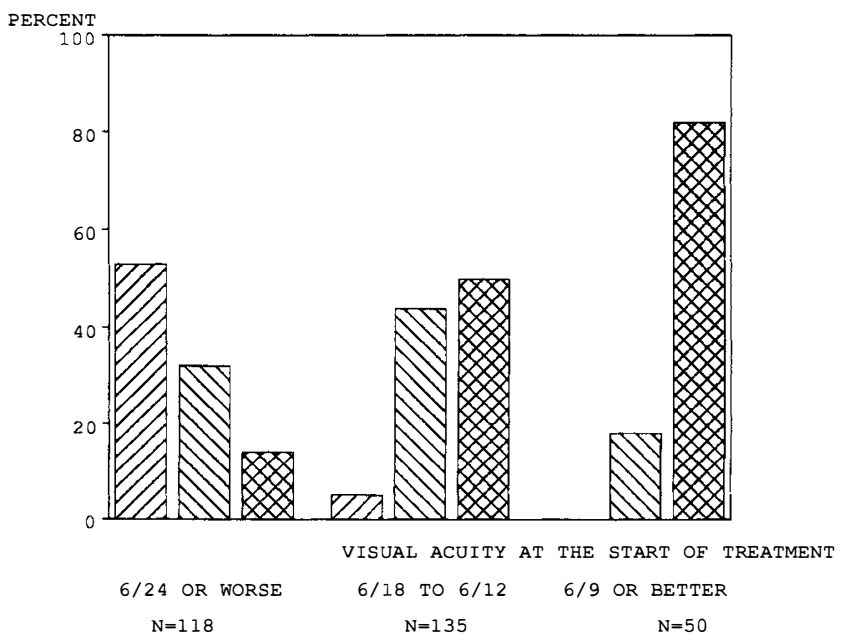

Fig. 3. The percentage of outcome of treatment according to visual acuity at the start of treatment. Cross hatching denotes $6 / 9$ or better, falling diagonal hatching denotes $6 / 12$ or $6 / 18$ and rising diagonal hatching denotes 6/24 or less.

outcome of treatment difficult. In young children it is often impossible to obtain linear visual acuities, as was the case in $52 \%$ of our sample. One hundred and twenty-six (34\%) of our patients were initially tested with single letters or pictures, a method of assessment which conceals the effect of the crowding phenomenon. ${ }^{8}$ Crowding is an important element of amblyopia, and so final visions were obtained by a linear method whenever possible. The discrepancies which occur between single optotypes and linear acuity because of the crowding phenomenon ${ }^{8}$ are varied and unpredictable, so it is not possible to adjust for it and obtain an equivalent linear vision for those patients tested with single optotypes.

It is likely that the results categorised by initial vision were affected by the crowding phenomenon as in $27 \%$ the initial vision was obtained with single optotypes and the final with a linear one. In some cases this may have given the appearance of deterioration during treatment. The effect of crowding almost certainly influenced the results in the group with 6/9 or better initial vision where, contrary to expectations, a successful outcome was observed in only $82 \%$ of children. It may also help to explain our rather disappointing result in the 6/18-6/12 initial vision group.

The age of starting treatment is believed to affect the outcome of treatment. ${ }^{9,10,11,12}$ Eibschitz et al presented a $^{9}$

Table IV. The outcome of treatment according to the reason for stopping occlusion

\begin{tabular}{|c|c|c|c|c|c|c|c|c|}
\hline \multirow{3}{*}{$\begin{array}{l}\text { Reason for } \\
\text { stopping } \\
\text { occlusion }\end{array}$} & \multirow{2}{*}{\multicolumn{2}{|c|}{$6 / 9$}} & \multicolumn{4}{|c|}{ Outcome } & \multirow{2}{*}{\multicolumn{2}{|c|}{$\begin{array}{c}\text { Missing } \\
\text { data }\end{array}$}} \\
\hline & & & $6 / 12-$ & $6 / 18$ & $\begin{array}{c}\text { less } \\
6 /\end{array}$ & $\begin{array}{l}\text { han } \\
8\end{array}$ & & \\
\hline & Nos & $\%$ & Nos & $\%$ & Nos & $\%$ & Nos & $\%$ \\
\hline $\begin{array}{l}\text { Instruction from } \\
\text { orthoptist }\end{array}$ & 125 & 42 & 96 & 32 & 59 & 20 & 16 & 5 \\
\hline $\begin{array}{l}\text { Non-compliance or } \\
\text { failure to attend }\end{array}$ & 10 & 18 & 18 & 32 & 21 & 37 & 8 & 14 \\
\hline Other & 2 & 13 & 6 & 40 & 5 & 33 & 2 & 13 \\
\hline Total & 137 & 37 & 120 & 33 & 85 & 23 & 26 & 7 \\
\hline
\end{tabular}

success rate of $65 \%$ in children detected through visual screening at $1-2.5$ years, diminishing to only $3 \%$ in the group where amblyopia was not detected until eight to nine years. Despite the fact that the majority of our patients were referred prior to school age, our results do not support this theory. We believe that this is because of the presentation of the different types of amblyopia at different ages, and their differing response to treatment. Younger patients present mainly with strabismic and strabismic with anisometropic amblyopia, and they do not have as successful an outcome as the anisometropic amblyopes who tend to present at a later age. Our finding of a fairly equal outcome at all ages is supported by Birnbaum $^{10}$ et al in their literature survey of 23 published amblyopia studies.

The amount and type of the occlusion are the major factors affecting outcome that are under the direct control of the clinician. There is considerable debate among clinicians about the effectiveness of different types and durations of occlusion, and good results have been reported from a number of methods. Watson et $\mathrm{l}^{12}$ compared conventional full-time and part-time occlusion, minimal occlusion and CAM treatment in two different populations and concluded that all of the methods are equally effective. Several methods of occlusion were adopted with the patients in our cohort but the vast majority received part-time total occlusion.

We have analysed our results based upon prescribed amounts of occlusion although it is probable that this exceeds the amount that was actually used. Our results show that the patients who received the lowest total number of hours of occlusion had the highest success rate, and that those who were prescribed a large total number of hours improved the least. When we looked at our results according to intensity (i.e. hours per day) and the duration (i.e.: in months) we found that the same situation applied, that those with lesser amounts of occlusion had the best outcome. In the series of amblyopes reviewed by Massie, ${ }^{5}$ those who failed to improve had more occlusion, and this seems to be the case in our series because the patients who

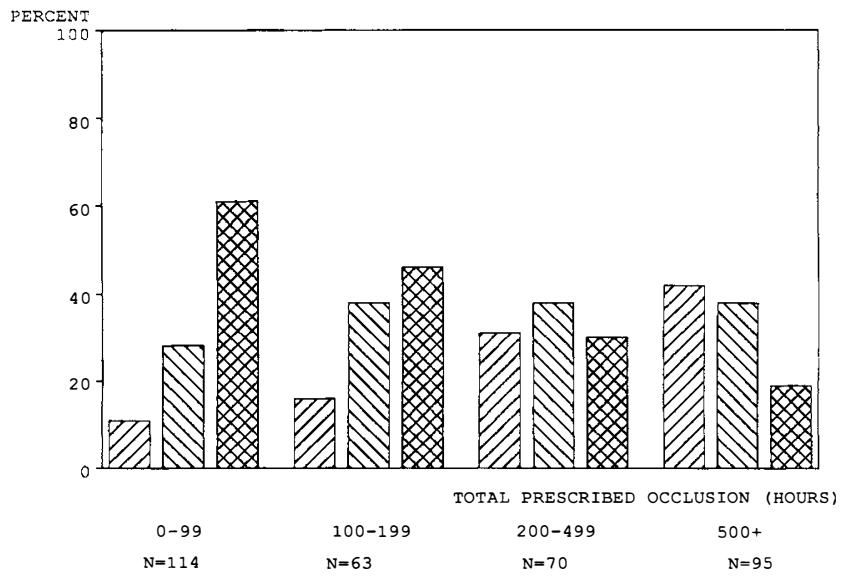

Fig. 4. The percentage outcome of treatment according to the total number of prescribed hours of occlusion. Cross hatching denotes $6 / 9$ or better, falling diagonal hatching denotes $6 / 12$ or $6 / 18$ and rising diagonal hatching denotes $6 / 24$ or less. 
failed to respond to their initial treatment were prescribed progressively more occlusion in the hope of achieving a success. It may be argued that a regime of full time total occlusion initially, expecially in those patients with poor initial visual acuity, might have led to more successful results with less amounts of occlusion overall. It is clear however that whatever the regime of treatment, there are a number of patients that will fail to respond to treatment regardless of how much occlusion they receive. We have not been able to identify any characteristics that might allow these patients to be recognised prior to treatment.

This study has provided a comprehensive survey of outcome of amblyopia treatment of unselected patients in one centre. We believe that further studies are needed of the outcome of treatment in other large centres.

We should like to thank the staff of the Orthoptic Department of Leicester Royal Infirmary for their cooperation with this study. Clerical assistance was provided by Ms K. Robinson and Ms R. Gowing, whose salary is supported by the Anne Allerton Fund.

Key words: Amblyopia, Occlusion, Survey.

\section{REFERENCES}

1. De Buffon GL: Sur le cause du strabisme ou des yeux louches. Hist Acad R Sci, 1743:231: cited by Ching et al, Practical management of amblyopia, J Paediat Ophthalmol Strabis 1986, 23: 12-16.
2. Flynn JT and Cassady JC: Currennt trends in amblyopia therapy. Ophthalmology 1978, 85: 428-50.

3. Scott WE and Dickey CF: Stability of visual acuity in amblyopic patients after visual maturity. Arch Clin Exp Ophthalmol 1988, 226: 154-7.

4. Thompson JR, Woodruff G, Hiscox FA, Strong N, Minshull $\mathrm{C}$ : The incidence and prevalence of amblyopia detected in childhood. Public Health (In press).

5. Massie H: Fixing Eye Occlusion: Survey of 1,000 cases of patients receiving occlusion of the fixing eye. Trans Ophthalmol Soc Aust 1965, 24: 39-46.

6. Bremner MH: Visual acuity in primary school children 4-12 years. Review of amblyopic treatment in this age group. Aust J of Ophthalmol 1984, 12: 395-9.

7. Lithander J and Sjöstrand J: Anisometopic and strabismic amblyopia in the age group 2 years and above: a prospective study of of the results of treatment. Br J Ophthalmol 1991, 75: $111-16$.

8. Von Noorden GK: Binocular Vision and Ocular Motility 4th Edition. CV Mosby, St Louis. 1990.

9. Eibschitz N, Friedman Z, Newmann E: Comparative results of amblyopia treatment. Metabol Ophthalmol 1978, 2: 111-112.

10. Birnbaum M, Koslowe K, Sanet R: Success in amblyopia therapy as a function of age, A literature survey. Am J Optom Physiol Opt 1977, 54: 269-75.

11. Oliver M, Neumann R, Chaimoritch, Gotesman N, Shimshoni M: Compliance and results of treatment for amblyopia in children more than 8 years old. Am J Ophthalmol 1986, 102: $340-5$.

12. Watson PG, Sanac AS, Pickering MS: A comparison of various methods of treatment of amblyopia. A block study. Trans Ophthalmol Soc UK 1985, 104: 319-28. 